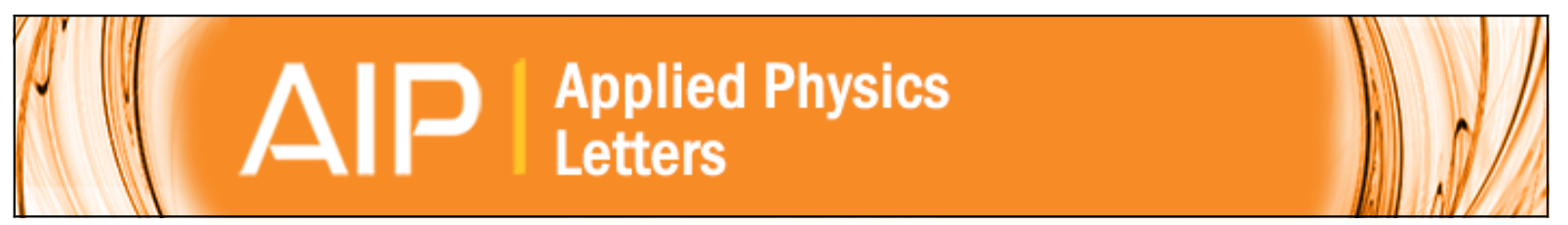

\title{
Ordered silicon nanocones arrays for label-free DNA quantitative analysis by surface- enhanced Raman spectroscopy
}

Ting-Ting Xu, Jian-An Huang, Li-Fang He, Yao He, Shao Su, and Shuit-Tong Lee

Citation: Applied Physics Letters 99, 153116 (2011); doi: 10.1063/1.3650937

View online: http://dx.doi.org/10.1063/1.3650937

View Table of Contents: http://scitation.aip.org/content/aip/journal/apl/99/15?ver=pdfcov

Published by the AIP Publishing

\section{Articles you may be interested in}

Surface-enhanced Raman spectroscopy study of single stranded DNA sequences on silver nanorod array AIP Conf. Proc. 1506, 53 (2012); 10.1063/1.4772525

Silicon-based reproducible and active surface-enhanced Raman scattering substrates for sensitive, specific, and multiplex DNA detection

Appl. Phys. Lett. 100, 203104 (2012); 10.1063/1.3701731

Silver vanadate nanoribbons: A label-free bioindicator in the conversion between human serum transferrin and apotransferrin via surface-enhanced Raman scattering

Appl. Phys. Lett. 98, 193110 (2011); 10.1063/1.3590712

A surface-enhanced Raman spectroscopy substrate for highly sensitive label-free immunoassay Appl. Phys. Lett. 92, 043116 (2008); 10.1063/1.2833695

Agarose-stabilized gold nanoparticles for surface-enhanced Raman spectroscopic detection of DNA nucleosides Appl. Phys. Lett. 88, 153114 (2006); 10.1063/1.2192573

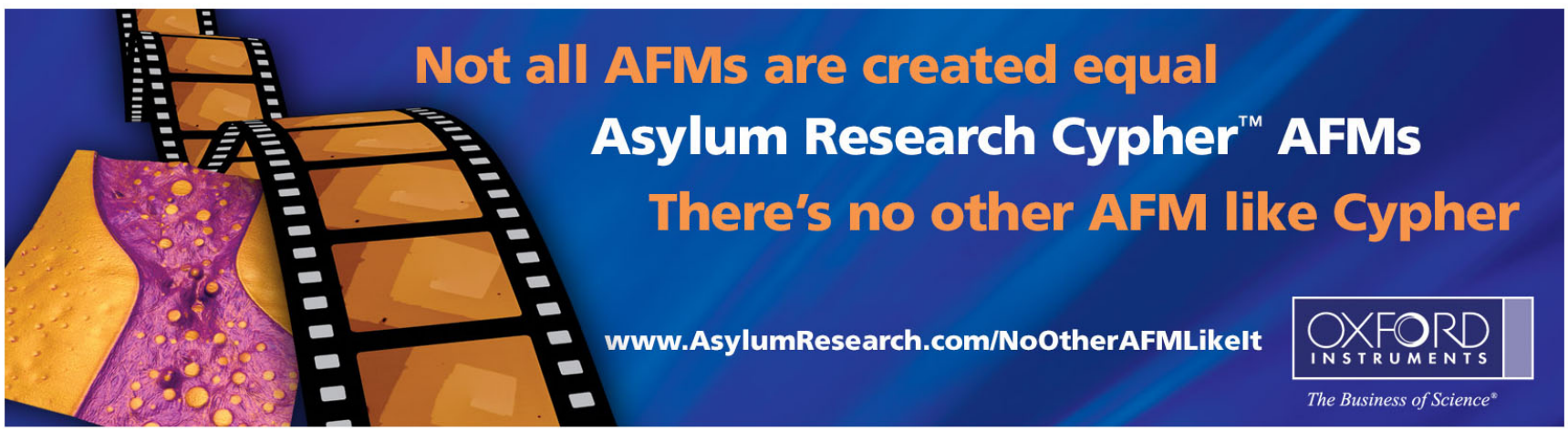




\title{
Ordered silicon nanocones arrays for label-free DNA quantitative analysis by surface-enhanced Raman spectroscopy
}

\author{
Ting-Ting $\mathrm{Xu},{ }^{1,2}$ Jian-An Huang, ${ }^{1} \mathrm{Li}$-Fang He,${ }^{1}$ Yao He,${ }^{2, a)}$ Shao $\mathrm{Su},{ }^{2}$ and Shuit-Tong Lee ${ }^{1, \mathrm{~b})}$ \\ ${ }^{1}$ Center of Super-Diamond and Advanced Films (COSDAF) and Department of Physics and Materials Science, \\ City University of Hong Kong, Hong Kong Special Administrative Region \\ ${ }^{2}$ Institute of Functional Nano \& Soft Materials (FUNSOM) and Jiangsu Key Laboratory for Carbon-Based \\ Functional Materials \& Devices, Soochow University, Suzhou, Jiangsu 215123, China
}

(Received 2 August 2011; accepted 14 September 2011; published online 14 October 2011)

\begin{abstract}
Ordered vertical silicon nanocones arrays coated with silver nanoparticles (AgNPs@SiNCs) are developed as surface-enhanced Raman scattering (SERS)-active substrate, which features good uniformity and reliable reproducibility of SERS signals. Label-free DNA at low concentrations $\left(10^{-8} \mathrm{M}\right)$ could be quantitatively analyzed via SERS using the AgNPs@SiNCs. The Raman peak at $732 \mathrm{~cm}^{-1}$ due to adenine breathing mode was selected as an endogenous Raman marker for quantitative detection of label-free DNA. The AgNPs@SiNCs as high-performance SERS-active substrates are attractive for surface enhancement mechanism investigation and biochemical sensing applications. (C) 2011 American Institute of Physics. [doi:10.1063/1.3650937]
\end{abstract}

Surface-enhanced Raman scattering (SERS) spectroscopy is well recognized as a powerful tool for ultrahighsensitivity detection of biological molecules, especially DNA. ${ }^{1,2}$ Various SERS-active substrates (e.g., silver or gold nanoparticles (NP)) have been developed for the detection of biomolecules at extremely low concentrations ( $\sim$ pico mole). ${ }^{3}$ Free-standing DNA molecules often have significantly smaller Raman scattering cross-section than fluorescent dyes, yielding weak Raman signals even at high concentrations $(\sim \mu \mathrm{mol}){ }^{4}$ For high-sensitivity detection, DNA is generally tacked with Raman signal reporters (e.g., fluorescent dyes) to produce amplified SERS signals. However, such modification manipulations are often expensive and complicated. Consequently, development of more practicable SERS substrates and detection strategies for label-free DNA detection continues to attract intense attention. A variety of SERS-active substrates with high enhancement factor and fast response at low excitation power to avoid DNA damage has been designed for sensitive detection of labelfree DNA. ${ }^{5,6}$ Despite the progress, uniform SERS-active substrates which can yield reproducible and strong SERS signals for label-free DNA detection are still in demand. ${ }^{7}$

Recently, silicon nanostructures (e.g., silicon nanowires (SiNWs), nanopillars, etc.) have been demonstrated to be highly effective SERS-active substrates due to their large surface-to-volume ratios and unique properties. ${ }^{8,9}$ In particular, our studies have revealed that SiNWs coated with silver nanoparticles feature high Raman enhancement factor and have been utilized for high-sensitivity detection of various chemi$\mathrm{cal} /$ biochemical species. $^{10,11}$ We recently shown that silicon nanopillars arrays could effectively confine the incident light on their surfaces, leading to improvement of light-conversion efficiency and enhancement of localized electromagnetic (EM) field. ${ }^{12}$ Based on those studies, we herein present AgNPs decorated-silicon nanocones arrays (AgNPs@SiNCs)

\footnotetext{
${ }^{\text {a)} E l e c t r o n i c ~ m a i l: ~ y a o h e @ s u d a . e d u . c n . ~}$

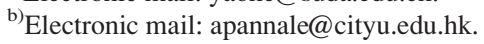

for high-performance SERS-active substrate. This substrate is highly uniform and ordered and can yield strong and reproducible SERS signals. Based on this SiNCs-based biosensor, label-free DNA of low concentration down to $10^{-8}$ mole can be rapidly and quantitatively detected.

Highly ordered SiNCs were obtained from prefabricated silicon nanopillar arrays, coated with AgNPs via ion-beam-sputtered deposition, as illustrated in Fig. 1(a). Patterned silicon nanopillars arrays were fabricated via nanosphere lithography and metal-induced etching [Fig. 1(b)] following our previous procedures. ${ }^{8,12}$ To enlarge the exposure area of the substrate to laser at normal incidence, silicon nanopillars were put in hydrogen fluoride solution (2\%) for 3 min and for another 5 min after adding silver nitrate solution $\left(10^{-4} \mathrm{M}\right)$ to obtain the cone-shape SiNCs. After removal of silver residues in nitric acid solution, the SiNCs were treated by ion beam sputtering of silver at an optimized sputtering time of $120 \mathrm{~s}$ under $12.5 \mathrm{~mA}$. Afterwards, each SiNC was completely decorated with AgNPs around $20 \mathrm{~nm}$ in diameters, as shown in Figs. 1(c) and 1(d). Elements of silicon, silver, oxygen, and carbon could be observed in the energydispersive x-ray spectroscopy of AgNPs@SiNCs before DNA assembly [Fig. 2(a)]. However, oxidization of SiNCs and AgNPs as well as carbon pollution of the system by the environment have little effect in DNA detection experiments. The system left in air condition more than two weeks showed no obvious decrease in surface enhancement performance. AgNPs@SiNCs were cut into small pieces of $5 \times 5 \mathrm{~mm}^{2}$ and washed 3 times by milli-Q water before DNA immobilization. Thiol-modified single-strand DNA (S1: $3^{\prime}$-HS- $(\mathrm{CH} 2)$ 6-TGAGTGGACGTCAACGAGCAA-5'; S2: 3'-HS-(CH2)6ATAACATTCCTCCGCAATACTCCAAAAGGTAATTA-5' was diluted to $10^{-6} \mathrm{M}, 10^{-7} \mathrm{M}, 10^{-8} \mathrm{M}$ with sterile water; SiNCs were immersed in the incubation solution over-night for DNA immobilization. ${ }^{11}$ The as-prepared sample was washed carefully in milli-Q water, followed by drying in nitrogen flow to avoid physisorption (or non-specific binding) of chemicals on the substrates. No fluorescent labels or Raman dyes were used. 

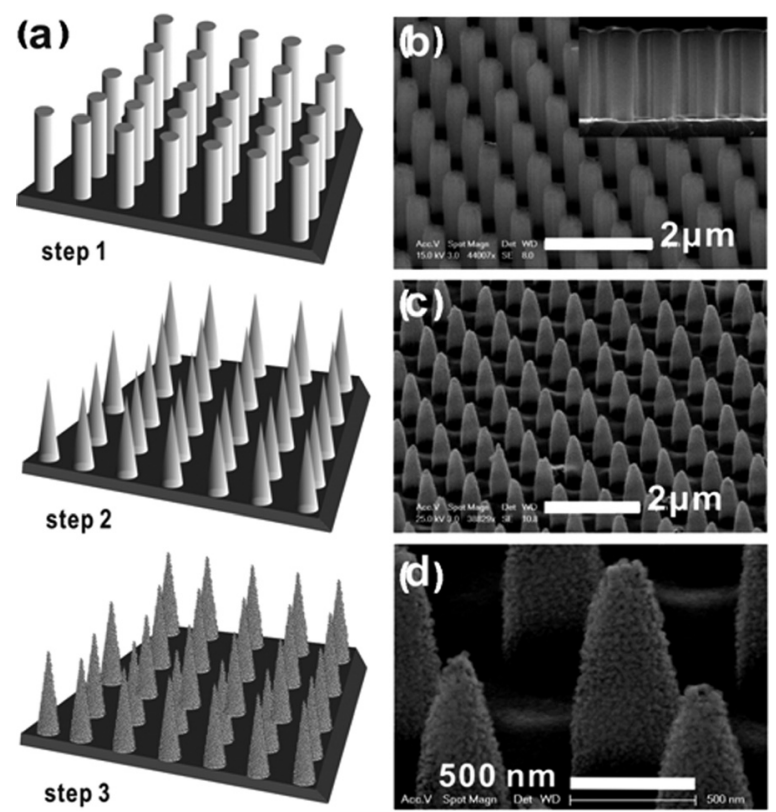

FIG. 1. (a) Schematic of fabrication procedures of AgNPs@SiNCs from silicon nanopillars arrays template. (b) Typical SEM image of the silicon nanopillars array template obtained following (a). The inset shows the cross-section view of the array. (c) Silicon nanocones array after silver nanoparticles sputtered for 2 min over the body via IBSD system, following step 3 (average bottom diameter $\sim 474 \mathrm{~nm}$, top diameter $\sim 100 \mathrm{~nm}$, and height $\sim 1 \mu \mathrm{m}$ ). (d) High-magnification SEM of AgNPs@SiNCs showing AgNPs $10-30 \mathrm{~nm}$ in diameters.

Figure 1 shows the as-prepared vertical AgNPs@SiNCs are highly ordered with a uniform diameter and length. The inset in Fig. 2(b) shows colorful diffraction pattern of SiNCs under ambient light, revealing the highly periodic structure in macro scale. Reflectance spectra of AgNPs@SiNCs were investigated as a function of wavelength using a UV-Vis-IR absorption spectrometer. Fig. 2(b) shows the reflectance spectrum reaches the minimal value around $500 \mathrm{~nm}$, denoting the optimal resonant excitation. ${ }^{7}$ Importantly, the reflectance spectrum reveals over $90 \%$ of the incident energy is absorbed by the substrate, which greatly increases the surface enhancement efficiency. $514 \mathrm{~nm}$ was chosen as the excitation wavelength in the following Raman-relevant experiment to take advantage of the optimal resonant excitation and light coupling around $500 \mathrm{~nm}$.

Raman maps without biomolecules were conducted to evaluate the uniformity of the system. Optical-phonon peak of silicon at $520 \mathrm{~cm}^{-1}$ is the strongest silicon Raman band
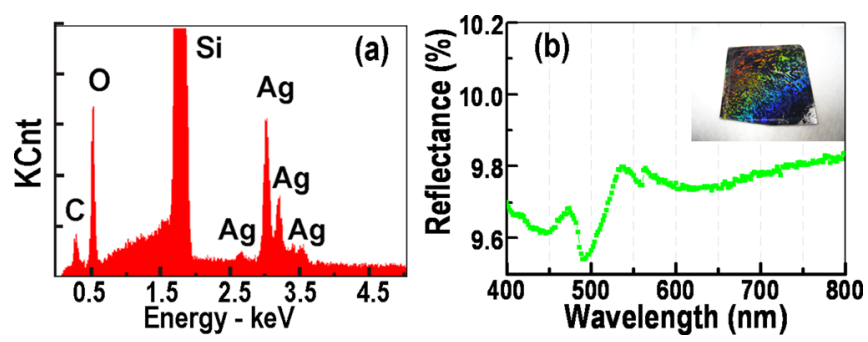

FIG. 2. (Color online) (a) EDAX characterization of AgNPs@SiNCs before assembly with DNA. (b) Reflectance spectrum of AgNPs@SiNCs at normal incidence. Inset illustrates the strong diffraction pattern of a $20 \mathrm{~mm} \times 20$ mm AgNPs@SiNCs wafer when illuminated by ambient light.
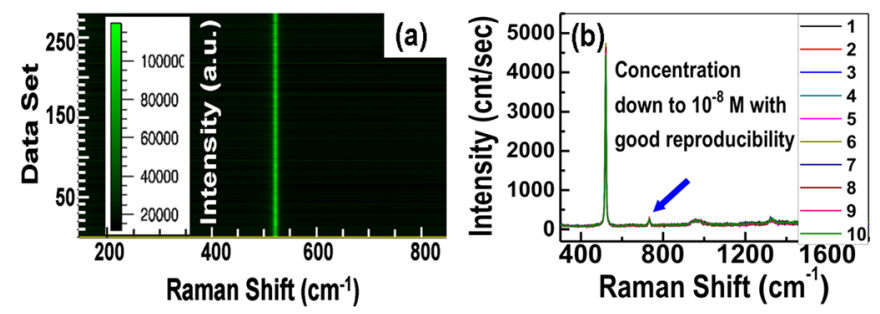

FIG. 3. (Color online) (a) Raman map without assembly with DNA. There are $\sim 280$ data sets in a map area $\sim 40 \times 40 \mu \mathrm{m}^{2}$. Color depth denotes peak intensity at that point. (b) Raman spectra of $10^{-8}$ M DNA S1 from 10 randomly selected locations on AgNPs@SiNCs recorded at 514 nm excitation with $\mathrm{P}=177 \mu \mathrm{W}$ and $\mathrm{T}=1 \mathrm{~s}$.

frequently used as the distinctive and standard reference in conventional Raman studies. ${ }^{13}$ Fig. 3(a) shows one example of the mapping results. The uniform peak intensities at 520 $\mathrm{cm}^{-1}$ from $\sim 280$ data sets demonstrate the uniformity of the AgNPs@SiNCs.AgNPs@SiNCs were further utilized as SERS substrates for label-free DNA analysis. The SERS spectra from the DNA samples are in well accord with literature reports, which are dominated by the strong peak at 732 $\mathrm{cm}^{-1} .^{14}$ This Raman peak, being adenine breathing mode and the most characteristic Raman peak of DNA, was selected as an endogenous Raman marker for label-free DNA detection. ${ }^{6}$ Fig. 3(b) shows SERS assays at 10 random points from SiNCs incubated with $10^{-8}$ M DNA (S1). Significantly, all 10 points produced nearly identical Raman spectra under same experiment conditions (laser power: 177 $\mu \mathrm{W}$; exposure time: $1 \mathrm{~s}$ ), demonstrating good reproducibility and fast response of Raman signals from the AgNPs@SiNCs for low concentration of DNA under low excitation power. Moreover, several Raman maps spectra from large-area AgNPs@SiNCs substrate $\left(50 \times 40 \quad \mu \mathrm{m}^{2}\right)$ show similar Raman intensity and spectra [Fig. 4(a) inset] for $10^{-6} \mathrm{M}$ DNA S1 with a laser power of $177 \mathrm{~W}$ and an exposure time of $0.1 \mathrm{~s}$ at each point. Peak intensities distribution of DNA and silicon was analyzed from exhaustive Raman map measurement results. As shown in Fig. 4(a), DNA peak at 732 $\mathrm{cm}^{-1}$ and silicon peak at $520 \mathrm{~cm}^{-1}$ both show narrow distribution capable for quantitative analysis; and the average intensity of DNA Raman peak is even higher than that of silicon.
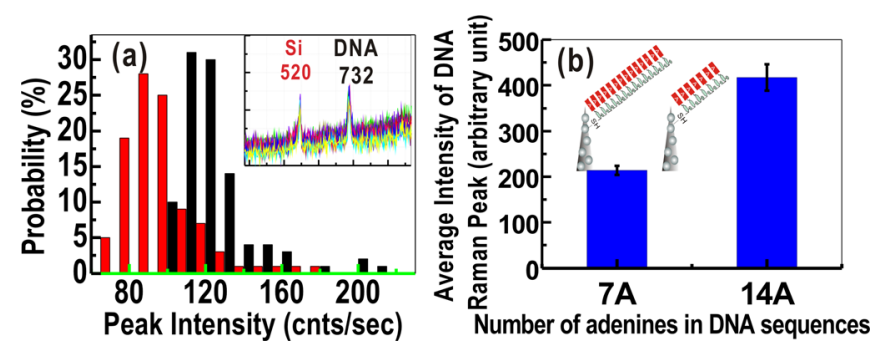

FIG. 4. (Color online) (a) Measured intensity probability distribution of $\mathrm{Si}$ $520 \mathrm{~cm}^{-1}$ and DNA $732 \mathrm{~cm}^{-1}$ Raman peak from SERS spectra of $10^{-6} \mathrm{M}$ DNA S1 on AgNPs@SiNCs substrate mapping from an area $50 \times 40 \mu \mathrm{m}^{2}$ using $514 \mathrm{~nm}$ excitation at $\mathrm{P}=177 \mu \mathrm{W}$ and $\mathrm{T}=0.1 \mathrm{~s}$. Inset shows several overlapping SERS spectra from Raman map result. (b) Average intensities of DNA peak at $732 \mathrm{~cm}^{-1}$ between DNA S1 (7 A) and S2 (14 A). Statistic results come from exhaustive measurements. Error bar stands for standard deviation of the data. 
We next applied AgNPs@SiNCs for quantitative analysis of label-free DNA with different number of adenines (A). The Raman peak intensity at $732 \mathrm{~cm}^{-1}$ of the DNA containing $14 \mathrm{~A}\left(\mathrm{~S} 210^{-8} \mathrm{M}\right)$ is nearly twice as intense as that of the DNA containing $7 \mathrm{~A}\left(\mathrm{~S} 10^{-8} \mathrm{M}\right)$. The statistic data from exhaustive measurements demonstrated a direct ratio relationship between the average peak intensities and number of adenines in DNA sequences, as indicated by Fig. 4(b) with small error bars. Compared with traditional methods for quantitative analysis of DNA, such as polymerase chain reaction (PCR), the present Raman technique could get results more quickly and at less cost. ${ }^{15}$

The present system combines the advantages of topdown and bottom-up technologies to achieve both uniformity and high SERS enhancement, which enables the quantitative analysis of label-free DNA. Due to morphology improvement, we suppose the excellent Raman enhancement properties of AgNPs@SiNCs would be mainly ascribed to two aspects, leading to distinctive enhancement mechanism of AgNPs@SiNCs versus published cone-shape SERS substrates. ${ }^{5,16}$ The highly ordered periodic structure of AgNPs@SiNCs would effectively attract and confine light inside and on the surface of SiNCs; thus by choosing wavelength of excitation light at optimal resonance point, the EMfield near the surface of AgNPs could be significantly magnified due to the resonance of Raman light. ${ }^{12}$ Meanwhile, the high packing density of AgNPs with diameters $\sim 20 \mathrm{~nm}$ on the three-dimensional surface of SiNCs enables the coupling of surface plasmon polaritons. Additionally, the ordered SiNCs arrays with rational interspaces would facilitate the efficient assembly of biomolecules into three-dimensional system, which would expand the detection workspace.

DNA was assembled on the surface of AgNPs via SHAg chemical bond. The Raman scattering cross-section of thiol-modified label-free DNA would be amplified prominently by the surrounding high EM-field near the surface of AgNPs. Meanwhile, Raman peak of adenine breathing mode shifts around $3 \mathrm{~cm}^{-1}$ compared to literature values, ${ }^{6,14}$ which may be caused by the interaction between single-strand DNA and AgNPs@SiNCs system.

To conclude, we prepare a silicon-based SERS-active substrate, i.e., AgNPs-coated SiNCs via a top-down method. The resultant AgNPs@SiNCs is highly uniform and features excellent SERS properties, including high enhancement and reproducibility of Raman signals. Label-free DNA of low concentrations $\left(10^{-8} \mathrm{M}\right)$ could be quantitatively detected by using the AgNPs@SiNCs. AgNPs@SiNCs as highperformance SERS-active substrate is promising various quantitative chemical and biochemical sensing applications. The highly ordered AgNPs@SiNCs provides a good platform to investigate the EM-field enhancement mechanism of SERS as well as light propagation and localization phenomenon in periodic matrix structures.

This paper is supported by Research Grants Council of HKSAR (No. CityU5/CRF/08) and RGC-NSFC Joint research scheme (No. N_CityU 108/08) NSFC (30900338, 51072126), and National Basic Research Program of China (973 Program) 2012CB932400.

${ }^{1}$ J. Kneipp, H. Kneipp, and K. Kneipp, Chem. Soc. Rev. 37, 1052 (2008); D. Graham and R. Goodacre, Chem. Soc. Rev. 37, 883 (2008); T. VoDinh, H.-N. Wang, and J. Scaffidi, J. Biophoton. 3, 89 (2009).

${ }^{2}$ J. Wang, Nucleic Acids Res. 28, 3011 (2000); P. O. Brown and D. Botstein, Nat. Genet. Suppl. 21, 33 (1999).

${ }^{3}$ K. Faulds, W. E. Smith, and D. Graham, Anal. Chem. 76, 412 (2004); R. J. Stokes, A. Macaskill, P. J. Lundahl, W. E. Smith, K. Faulds, and D. Graham, Small 3, 1593 (2007).

${ }^{4}$ S. A. Meyer, E. C. L. Ru, and P. G. Etchegoin, J. Phys. Chem. A 114, 5515-5519 (2010); S. Shim, C. M. Stuart, and R. A. Mathies, Chem. Phys. Chem. 9, 697 (2008).

${ }^{5}$ H.-C. Lo, H.-I. Hsiung, S. Chattopadhyay, H.-C. Han, C.-F. Chen, J. P. Leu, K.-H. Chen, and L.-C. Chen, Biosens. Bioelectron. 26, 2413 (2011).

${ }^{6}$ A. Barhoumi and N. J. Halas, J. Am. Chem. Soc. 132, 12792 (2010).

${ }^{7}$ X. G. Deng, G. B. Braun, S. Liu, P. F. Sciortino, J. B. Koefer, T. Tombler, and M. Moskovits, Nano Lett. 10, 1780 (2010).

${ }^{8}$ K. Q. Peng, M. L. Zhang, A. J. Lu, N.-B. Wong, R. Q. Zhang, and S.-T. Lee, Appl. Phys. Lett. 90, 163123 (2007).

${ }^{9}$ M. W. Shao, D. D. D. Ma, and S.-T. Lee, Eur. J. Inorg. Chem. 27, 4264 (2010); Y. He, C. H. Fan, and S.-T. Lee, Nano Today 5, 282 (2010).

${ }^{10}$ M.-W. Shao, M.-L. Zhang, N.-B. Wong, D. D. D. Ma, H. Wang, W. W. Chen, and S.-T. Lee, Appl. Phys. Lett. 93, 233118 (2008); M.-L. Zhang, X. Fan, H.-W. Zhou, M.-W. Shao, J. A. Zapien, N.-B. Wong, and S.-T. Lee, J. Phys. Chem. C 114, 1969 (2010).

${ }^{11}$ Y. He, S. Su, T. T. Xu, Y. L. Zhong, J. A. Zapien, J. Li, C. H. Fan, and S.-T. Lee, Nano Today 6, 122 (2011).

${ }^{12}$ J. A. Huang, Y. Q. Zhao, X. J. Zhang, L. B. Luo, Y. K. Liu, J. A. Zapien, C. Surya, and S.-T. Lee, Appl. Phys. Lett. 98, 183108 (2011).

${ }^{13}$ M. Yang, D. M. Huang, P. H. Hao, F. L. Zhang, X. Y. Hou, and X. Wang, J. Appl. Phys. 75, 651 (1994); D. Nesheva, C. Raptis, I. Bineva, Z. Aneva, and Z. Levi, J. Appl. Phys. 92, 4678 (2002).

${ }^{14}$ A. Barhoumi, D. M. Zhang, F. Tam, and N. J. Halas, J. Am. Chem. Soc. 130, 5523 (2008).

${ }^{15}$ Y. M. D. Lo, M. S. C. Tein, T. K. Lau, C. J. Haines, T. N. Leung, P. M. K. Poon, J. S. Wainscoat, P. J. Johnson, A. M. Z. Chang, and N. M. Hjelm, Am. J. Hum. Genet. 62, 768 (1998).

${ }^{16}$ J.-P. Coppe, Z. D. Xu, Y. Chen, and G. L. Liu, Nanotechnology 22, 245710 (2011). 